Santa Clara University

Scholar Commons

University Library

Information Services

$10-31-2011$

\title{
Continual Evolution: The Experience Over Three Semesters of a Librarian Embedded in an Online Evidence-Based Medicine Course for Physician Assistant Students
}

Shannon Kealey

Santa Clara University, skealey@scu.edu

Follow this and additional works at: http://scholarcommons.scu.edu/library

Part of the Information Literacy Commons, and the Medical Education Commons

\section{Recommended Citation}

Kealey, Shannon. "Continual Evolution: The Experience Over Three Semesters of a Librarian Embedded in an Online Evidence-Based Medicine Course for Physician Assistant Students." Medical Reference Services Quarterly 30.4 (2011): 411-25.

This is an Accepted Manuscript of an article published by Taylor \& Francis in Medical Reference Services Quarterly on Oct. 31, 2011, available online: http://www.tandfonline.com/10.1080/02763869.2011.609046.

This Article is brought to you for free and open access by the Information Services at Scholar Commons. It has been accepted for inclusion in University Library by an authorized administrator of Scholar Commons. For more information, please contact rscroggin@scu.edu. 


\title{
Continual Evolution: The Experience Over Three Semesters of a Librarian Embedded in an Online Evidence-Based Medicine Course for Physician Assistant Students
}

\author{
SHANNON KEALEY \\ Henry Birnbaum Library, Pace University, New York, New York
}

This column examines the experience, over three years, of a librarian embedded in an online Epidemiology and Evidence-based Medicine course, which is a requirement for students pursing a Master of Science in Physician Assistant Studies at Pace University. Student learning outcomes were determined, a video lecture was created, and student learning was assessed via a five-point test during year one. For years two and three, the course instructor asked the librarian to be responsible for two weeks of the course instruction and a total of 15 out of 100 possible points for the course. This gave the librarian flexibility to measure additional outcomes and gather more in-depth assessment data. The librarian then used the assessment data to target areas for improvement in the lessons and Blackboard tests. Revisions made by the librarian positively affected student achievement of learning outcomes, as measured by the assessment conducted the subsequent semester. Plans for further changes are also discussed.

\section{INTRODUCTION}

Pace University is a private institution with campuses in the New York Metropolitan area. Pace's College of Health Professions offers a Master of Science in Physician Assistant Studies (MSPAS) in partnership with Lenox Hill Hospital. The Pace University-Lenox Hill Hospital MSPAS is fully accredited by the Accreditation Review Commission on Education for the Physician Assistant (ARC-PA) and is a member of the Physician Assistant Education Association (PAEA). According to ARCPA Standard B2.10, "program curriculum must include instruction to prepare students to search, interpret, and evaluate the medical literature, including its application to individualized patient care." ${ }^{1}$ Evidence-based Medicine (EBM) is defined as "the conscientious, explicit, and judicious use of current best evidence in making decisions about the care of individual patients."2 Therefore, all PAS MSPAS candidates are required, in their third semester of study, to take PAS 514Epidemiology and Evidence-based Medicine.

PAS 514 is offered online via Blackboard. Prior to the spring 2009 semester, a librarian at Pace's Henry Birnbaum Library on the New York City campus was invited to be the instructor for the week of the course dealing with searching the

${ }^{1}$ Accreditation Review Commission on Education for the Physician Assistant, Accreditation Standards for Physician Assistant Education, 4th ed. (Johns Creek, GA: ARC-PA, 2010), http://www.arcpa.org/documents/Standards4thedititionFINALwithclarifyingchangesJuly2010.pdf. ${ }^{2}$ David L. Sackett et al., "Evidence Based Medicine: What It Is and What It Isn't," Bmj 312, no. 7023 (1996): 71-72. 
specialized health sciences databases using the principles of EBM. For the 2010 and 2011 semesters, the librarian was asked to be responsible for two weeks of instruction and a total of 15 out of 100 course points. This column describes the evolution of the librarian's role in the PAS-514 course, including changes in the lessons and assessments made after reflecting on the student assessment data, resulting in improvements in student achievement of learning goals, and plans for additional changes for the 2012 semester.

YEAR ONE-SPRING 2009

Planning the Lesson

The librarian and course instructor decided on four learning goals for the week of the librarian's instruction. It was a greed that the MSPAS students should:

1. Be able to identify key databases for any clinical question

2. Be able to use MeSH and CINAHL headings

3. Be able to create an effective search strategy in MEDLINE/CINAHL/Cochrane Library

4. Understand how the levels of biomedical evidence correspond to publication types in databases, and know how to target the highest quality articles

The course was designed so that, for each learning goal there would be a learning outcome assigned.

\section{LEARNING GOAL 1}

In order to meet Learning Goal 1, it was determined that the students should be A) able to identify MEDLINE, CINAHL, and Cochrane as the minimum three databases to search for any clinical question, and be able to identify PsycINFO as an additional database to search for clinical questions with psychosocial aspects.

\section{LEARNING GOAL 2}

There were three learning outcomes assigned to Learning Goal 2. The fist was that students B) should be able to take a keyword entry term and find the corresponding $\mathrm{MeSH}$ and CINAHL headings. The second was that students should C) know how to check "Details" after every PubMed search to determine whether automatic mapping to MeSH had been successful. The third was to be able to D) use the MeSH or CINAHL heading tree to see which narrower headings could be included if one were to "Explode" a subject heading.

\section{LEARNING GOAL 3}

The learning outcome associated with Goal 3 was that the student should be able to E) combine the MeSH or CINAHL headings and/or keywords using the appropriate Boolean operators (AND, OR).

\section{LEARNING GOAL 4}

The learning outcome associated with Goal 4 was that students should F) know which publication types in MEDLINE, CINAHL, and the Cochrane Library would 
correspond to each level of biomedical evidence. The second learning outcome for goal 4 was that students should be able to G) use limits in the MEDLINE and CINAHL databases to target the various publication types.

Creating the Tutorial

The video screen capture software Camtasia was employed to create the video lecture. The librarian decided to include all concepts of the lecture in one video, called "Searching the Literature: From Clinical Scenario to Best Evidence." Student attention span was a concern in the planning and production of the video. Based on student feedback shared by librarians at Boston University Medical Center who had chosen not to use a 60-minute tutorial covering similar concepts in one shot in favor of shorter video segments, ${ }^{3}$ the "Searching the Literature" video was kept as short as possible. In the planning stages, the goal was to keep the video under 15 minutes, but in order to include the information essential for the students to meet every learning goal, the final video was more than 27 minutes long.

The "Searching the Literature" video began with a clinical question in PICO format, and demonstrated how to find MeSH and CINAHL headings. Entry terms such as "UTI" and "heart attack" were used to show the importance of finding and using the appropriate MeSH and CINAHL headings rather than relying on keyword searching alone. The "Details" box in PubMed and the "Suggest Subject Terms" option in EBSCO's CINAHL were emphasized. Students were shown how to check the MeSH tree in PubMed and the heading tree in CINAHL to decide whether to "Explode" a heading to include narrower headings. The demonstration included combining the headings with the appropriate Boolean operators in MEDLINE via PubMed, CINAHL via EBSCO, and the Cochrane Library. A "Levels of Evidence" pyramid was used to show the hierarchy of biomedical information, and corresponding publication types for MEDLINE and CINAHL were listed. The librarian also included a demonstration of how to use the Article Linker software to determine whether the student had access to a particular result from a search and how to use Interlibrary Loan (ILLiad) to request any articles not included in Pace subscriptions. The video is available for viewing at $<$ http://videoserv.pace.edu/library/ebm.wmv>.

\section{Challenges during Production}

In the final stages of production, the files size of the Camtasia project had grown so large that it continually caused computer-crashing problems. The final project took over an hour to render after several failed attempts due to computer crashes. The tendency for projects to crash the computer meant that making edits to the video would be extremely time consuming and difficult. In two sections of the lecture, the audio from a later point in the video fades in and out, over the correct audio for that

${ }^{3}$ Keven M. Jeffery, Lauren Maggio, and Mary Blanchard, "Making Generic Tutorials Content Specific: Recycling Evidence-Based Practice (EBP) Tutorials for Two Disciplines," Medical Reference Services Quarterly 28, no. 1 (2009): 1-9. 
section. It was impossible to edit out the "phantom audio" because it did not appear on the production timeline; there was nothing to delete. This glitch, possibly due to the large file size as well, remains in the finished product. Due to these challenges, future renditions of the tutorial will be broken up into a set of shorter videos.

The Five-Point Assessment

A Five-Point Blackboard test (see Appendix A) was created to assess learning outcomes. Five questions were worth one point each: three fill-in-the-blanks, one multiple choice, and one ordering. Because there were more learning outcomes than points on the test, not all learning outcomes could be measured. Learning outcomes A, B, C, D, and F were measured, but outcomes E and G were not. Of the 45 students enrolled in the course, 44 attempted and completed the assessment. The average score of those 44 students was 4.96 out of 5 points. There were so few mistakes that there was no recognizable trend or pattern.

Reflections after Year One

Reflecting on student achievement of learning outcomes and modifying course materials and assessments to improve student learning are integral parts of instruction for the benefit of the students as well as the pedagogical skills of the instructor. ${ }^{4}$ The course instructor informed the librarian that the students gave positive feedback regarding the video lecture in their course evaluations, although some students felt the pace of the video was too rapid. Student performance on the five-point quiz had been excellent, but because students had not been tested on some of the learning outcomes, for the following semester the course instructor and librarian agreed that the librarian's role would increase to two weeks of the course instruction and a total of 15 out of the 100 possible points for the course. This would allow the students to be introduced to additional information and test student learning outcomes $\mathrm{E}$ and $\mathrm{G}$.

\section{YEAR TWO-SPRING 2010}

Updating the Learning Materials

Because of the changes to both the PubMed and EBSCO interfaces to the MEDLINE and CINAHL databases, and a launch of a new library website, the video lecture produced in 2009 was almost immediately out of date. Production on a set of updated and more slowly paced videos to replace the 27-minute "Searching the Literature" video was begun but aborted due to limited production time caused by the need to produce additional lessons for week two. For spring 2010 semester, the

\footnotetext{
${ }^{4}$ Megan Oakleaf, "The Information Literacy Instruction Assessment Cycle: A Guide for Increasing Student Learning and Improving Librarian Instructional Skills," Journal of Documentation 65, no. 4 (2009): 539-60.
} 
same "Searching the Literature" video was used, supplemented with handouts showing screenshots of the interface changes.

The second weeks' lecture consisted of a Camtasia video on using the Clinical Queries filters in both MEDLINE via PubMed and CINAHL via EBSCO. This video was much shorter, only seven minutes. It contained an explanation of the Clinical Queries filters and a description of the search terms in the PubMed filters. EBSCO does not make this information publicly available, but the students were shown where the filters were located. The lecture tied into Learning Goal 4, as it would help students to quickly target high quality articles appropriate for their clinical questions. A new learning outcome was added. That is, students should $\mathrm{H}$ ) know how to use Clinical Queries in both the PubMed interface to MEDLINE and the EBSCO interface to both MEDLINE and CINAHL.

Assessment for Spring 2010

Because the 2009 data showed that a majority of the students were successful in achieving learning outcomes A-D and F after watching the "Searching the Literature" video, the librarian decided not to change the five-point assessment. For spring 2010 semester, the average grade on the week-one five-point assessment for the 52 students enrolled in the course was 4.94. Again, there were few student errors and no identifiable patterns.

For week two, students completed a new cumulative assessment tool worth the ten remaining points. For the cumulative ten-pint assessment, a Blackboard test was created with mostly essay responses where students would copy and paste their search histories for grading (see Appendix B). Learning outcomes B, C, E, G, and $\mathrm{H}$ were measured. The student responses to the cumulative ten-point assessment were collected, graded, and coded for common errors. The average grade was a respectable 9.17 out of ten, but the coding of the assessment data revealed two very common gaps in student achievement of the learning objectives. Thirty-one percent of the students made one or more mistakes that demonstrated a shallow understanding of Clinical Queries filters. Twenty-nine percent of the students made one or more mistakes that indicated a shallow understanding of MeSH. Very few students (fewer than 5\%) had trouble with formulating a search or using the proper Boolean operators, or other times of common search mistakes.

To address the gaps in student achievement indicated by the student assessment data, the course instructor was informed of the areas of weakness and an optional in-person training session was scheduled. The session was promoted ot the class through Blackboard and personal invitations were sent to the students whose errors fell into the two common categories. Only two of the 18 students contacted expressed interest in the training, so one-on-one meetings were held instead.

\section{Reflections and Changes after Year Two}

The two areas of the lessons and/or assessments that clearly needed revision were the sections having to do with using Clinical Queries and understanding MeSH. The 
most common mistake that indicated a shallow understanding of Clinical Queries was that the students left on their publication type limits from an earlier question, which interfered with their ability to see the difference between a sensitive and specific search. A student who fully understood the purpose of Clinical Queries would not make this error. Making this error would also prevent a student from better understanding Clinical Queries by observing the results of a sensitive Clinical Queries search as opposed to a specific one. Since the Clinical Queries video clearly showed the filters generated by each type of PubMed Clinical Query, the librarian reminded the students in the week-two cumulative ten-point assessment instructions to turn off limits for the Clinical Queries searches, allowing them to see the difference in results between sensitive and specific searches.

Although no students missed the questions ion the week-one five-point assessment having to do with checking "Details" or mapping to a MeSH heading, the most common mistake of this type made in the week-two cumulative 10-point assessment was that the student had not found and used the MeSH heading "insulin infusion systems" for the entry term "insulin pump." Like the example of "UTI" in the "Searching the Literature" video, the entry term "insulin pump" does not automatically map to the appropriate MeSH heading. It was planned to emphasize the importance of "Details" and searching MeSH directly even more distinctly in the updated lesson.

\section{YEAR THREE-SPRING 2011}

Production on a set of three slower-paced videos to replace the "Searching the Literature" video for the 2011 semester began in December 2010. However, one week before the spring 2011 semester started, there were additional changes to the PubMed interfaces, so the librarian aborted the update and created new supplemental handouts with screen shots of the interface changes. The original 2009 video was used once again so the students would understand the concepts necessary to reach the goals and outcomes. Additional links to PubMed videos produced by the National Library of Medicine were added to the course assignments on Blackboard so students would familiarize themselves with the new interface and gain a more in-depth understanding of how to use the MeSH database. National Library of Medicine PubMed videos linked from Blackboard included: PubMed Simple Subject Search, Searching with the MeSH database, and Combining MeSH terms using the MeSH database.

Assessment for Spring 2011

Student performance on the week-one five-point assessment was even better than the previous two semesters. The average score for the 53 students enrolled in the course was 4.98 out of 5 . There were only two one-point student errors and no discernable patterns.

Student performance on the week-two cumulative ten-point assessment was also very good, with an average of nine out of ten. Most notable was the marked decrease in student errors that showed a shallow understanding of Clinical Queries. 
Additional links to PubMed videos 5 produced by the National Library of Medicine were added to the course assignments on Blackboard so students would familiarize themselves with the new interface and gain more in-depth understanding of how to use the MeSH database. Whereas 31\% of students in 2010 had made an error of this type, only $3.7 \%$ made such an error in the spring of 2011 (see Figure 1). The additional instructions reminding students that publication type limits would interfere with a Clinical Queries search resulted in fewer student errors; what still was not clear was whether students grasped the purpose of Clinical Queries in general.

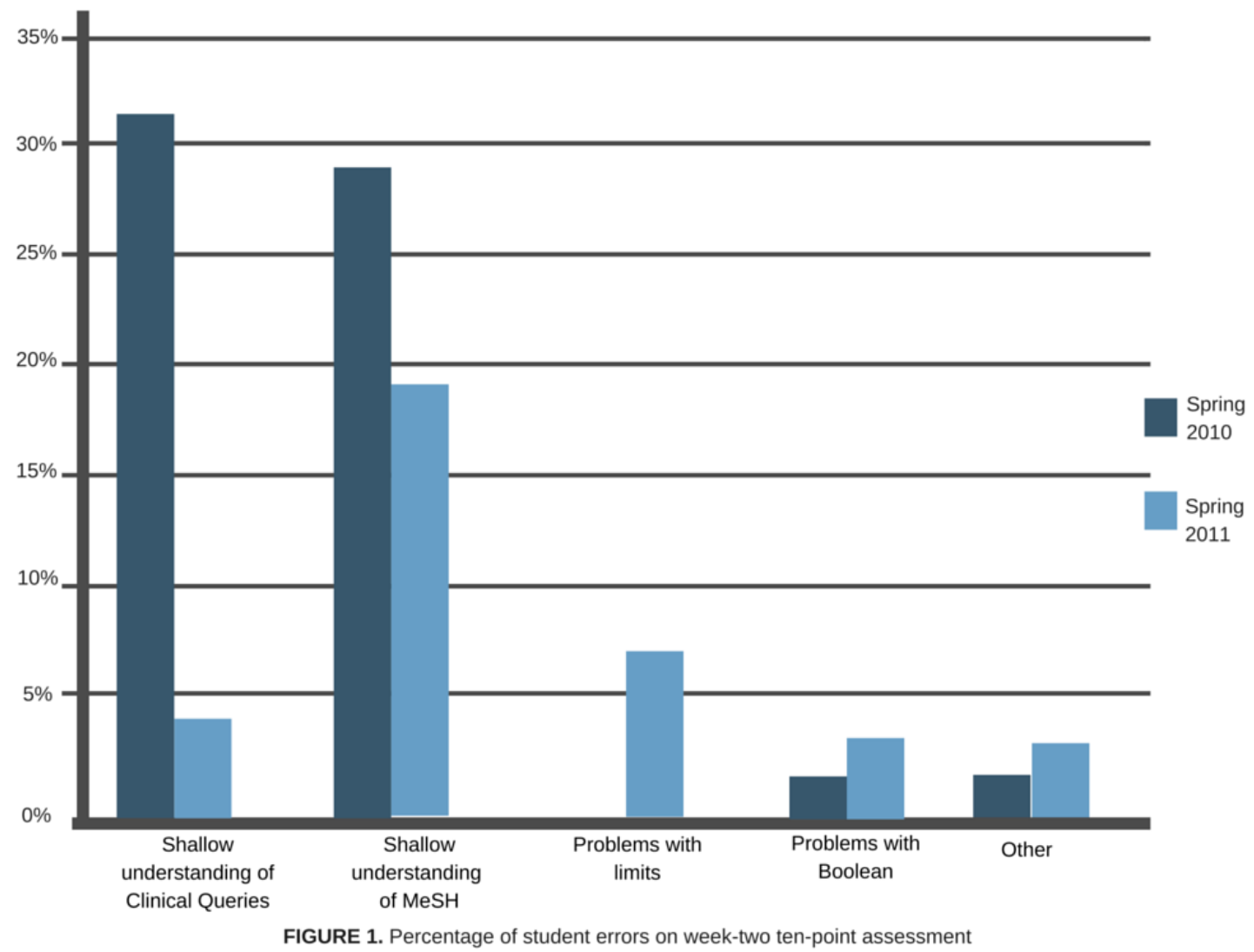

Errors in which students had trouble finding a MeSH heading for one or more elements of the clinical question in Questions 2, 4, 5, or 6 (see Appendix B) were coded as indicating a shallow understanding of MeSH. Although this decrease in these errors was not as dramatic, the percentage of students who made such an error fell from 29\% in 2010 to $18.9 \%$ in 2011. Another area of weakness also emerged in 2011: four (7.5\%) of the students either forgot to add their limits or applied the limits to the entire database rather than to their search result in Question 2 (see Appendix B).

${ }^{5}$ National Library of Medicine, "PubMed Online Training," accessed July 13, 2011, http://www.nlm.nih.gov/bsd/disted/pubmed.html. 


\section{Reflections and Plans for Year Four}

Because it is unclear whether the decrease in student errors regarding Clinical Queries indicates a better understanding of what the filters do, the methodology filters will be explained in more depth in the updated Clinical Queries video, and a question that requires students to articulate the function of the filters will be added to the week-two cumulative ten-point assessment.

To make room on the ten-point assessment for this new question, Question 1 will be converted into instructions. On reflection of the purpose of Question 1, which had been to set up the rest of the questions on the assessment rather than to test knowledge of one of the learning outcomes, it makes more sense to provide the students with the clinical question in PICO format, especially since the ability of students to put a clinical question into PICO format was measured by the course instructor in a previous week of the class.

Because the week-two cumulative ten-point assessment requires students to find a MeSH heading that is not found through automatic mapping in PubMed, students continue to have problems, although fever of them. In the new set of videos, the importance of checking "Details" in PubMed will be emphasized even more clearly. Also, the instructions for Question 2 in the week-two cumulative tenpoint assessment will include a reminder to check "Details" after every search.

Student ability to use Article Linker to find full text and student knowledge of Interlibrary Loan (ILLiad) were not measured through either assessment tool. Initially, the five-point assessment associated with the "Searching the Literature" video was to be the only assessment for the librarian's lessons, so including those skills as desired goals or outcomes was outranked by the need to test students on outcomes A-H. However, learning outcomes B and C were more effectively measured by the week-two ten-point cumulative assessment, rendering some of the questions on the week-one test unnecessary. This is an excellent opportunity to modify the week-one assessment to test student understanding of the Article Linker software and ILLiad.

The careful analysis of student assessment data in comparison to the learning outcomes and goals for the two weeks of librarian instruction has allowed for continual improvement in teaching materials, learning assessments, and librarian pedagogical skills. Continued partnership with the PAS 514 course instructor ensures that student achievement of the desired learning outcomes will improve as well.

\section{APPENDIX A: FIVE-POINT WEEK-ONE ASSESSMENT}

\section{Instructions}

You will have three (3) attempts to complete this quiz. I will be personally grading each one, so if you miss a question due to a typo, I will make sure you get credit.

Total Points: 5

Total Questions: 5 
Medium: Blackboard

Question 1 (Fill in the blank)

From the Pace Library Homepage, go to the PubMed database. Run the following search: Lou Gehrig's disease. What do you check to make sure that your search term mapped to the correct MeSH heading?

Correct Answer: Details OR Search Details

Feedback for Incorrect Answer

The correct answer is Details. After every PubMed search, you should check Details to see if your entry term mapped to the appropriate MeSH term.

Question 2 (Fill in the blank)

What is the MeSH term for our entry term, "Lou Gehrig's Disease"? (Hint: Check Details or the MeSH database - either will work.)

Correct Answer: amyotrophic lateral sclerosis

Feedback for Incorrect Answer

The correct answer is amyotrophic lateral sclerosis. If you missed this due to a typo, I will make sure you get credit. Remember, if your entry term does not map correctly, you should search the $\mathrm{MeSH}$ database for the correct term

Question 3 (Multiple Choice)

The PubMed interface to MEDLINE automatically Explodes MeSH terms to include the narrower headings indented underneath them, but EBSCO does not do that for the CINAHL and Medline databases. Select the answer below that matches one of the narrower Subject Headings that would be included if you Exploded the term "Diabetes Mellitus" in CINAHL.

Correct Answer: Diabetes Mellitus, Type 1 (Other Choices offered were: Diabetes Insipidus; Hyperglycemia; Endocrine Diseases)

Feedback for Incorrect Answer

The correct answer is Diabetes Mellitus, Insulin-Dependent, which is a narrower Subject Heading indented underneath the Subject Heading "Diabetes Mellitus." If you were to click "Explode" for the term "Diabetes Mellitus," this and all the other narrower subject headings indented underneath it would be included in the search.

Question 4 (Jumbled Sentence)

Correct Answer: According to the Levels of Evidence pyramid from the video, some article types are considered to be of better quality than others. Here is a list of some of those publication types: Clinical Trial, Case Study, Randomized Controlled Trial, Meta-Analysis. If you ranked them according to the pyramid, the highest quality article is: Meta-analysis, next is Randomized Controlled Trial, followed by Clinical trial, and last is Case study.

Feedback for Correct Answer

Great job! Remember that, for rare conditions and/or treatments, there may not be a MetaAnalysis or RCT available.

Feedback for Incorrect Answer

Having trouble ranking the publication types? Please review the pyramid included in the video.

The correct order, from highest to lowest, is: Meta-Analysis, RCT, Clinical Trial, Case Study. 
Question 5 (Fill in the blank)

When you are searching for information to help you answer a clinical question, you should search a minimum of three (3) biomedical databases. I demonstrated searching MEDLINE (via PubMed-another option is searching MEDLINE via Ebsco-you do NOT need to search both), CINAHL, and the Cochrane Library. Name a database you could search if your clinical question has psychosocial aspects.

Correct Answer: PsycINFO

\section{Feedback for Incorrect Answer}

The correct answer is PsycINFO, the premier source for international literature in psychology and related disciplines. It is maintained by the American Psychological Association, and contains articles on psychiatry, education, business, medicine, nursing, pharmacology, law, linguistics, and social work. If your clinical question has psychosocial aspects, you should definitely search PsycINFO in addition to MEDLINE, CINAHL and the Cochrane Library.

\section{APPENDIX B: WEEK-TWO CUMULATIVE TEN-POINT ASSESSMENT}

(This test was developed for the spring 2010 semester. Question instructions between sets of asterisks were added to address gaps in student learning outcomes for the spring 2011 semester.)

\section{Description}

This is a 10-point quiz, which will build on what you learned in Module 5 and will evaluate your understanding of the Clinical Queries video. Be sure you have watched the Module 6 Video on Clinical Queries, $* * *$ and also have looked at the Module 6 Notes Document that will show you how to answer the questions on this quiz having to do with Clinical Queries.***

\section{Instructions}

Once you have launched this quiz, you will have 3 attempts to complete. I will correct grading for typos, etc.

For this quiz, you will be using the following clinical scenario:

Mary is a 14-year old patient with insulin dependent diabetes. She has had diabetes for 5 years, and has generally been compliant with traditional shot therapy, with an average A1C of 6.8. In the last year, her $\mathrm{A} 1 \mathrm{C}$ has ranged between 8.7 and 9.3. Adjustments to the amount of her basal and mealtime injections have not lowered her A1C significantly. You want to see if the evidence supports a switch to insulin pump therapy to better control her blood sugars and bring her A1C down.

Total Questions: 6

Total Points: 10

Medium: Blackboard

Question 1 (Essay-worth 1 point)

What is the clinical question for this scenario, in PICO format?

Answer Feedback

A response like this will receive full credit:

In a 14-year old patient with insulin-dependent diabetes mellitus, is pump therapy more effective than shot therapy for lowering $\mathrm{A} 1 \mathrm{C}$ ?

OR

In a 14-year old patient with poorly controlled insulin-dependent diabetes mellitus, is pump 
therapy more effective than shot therapy for lowering A1C?

Question 2 (Essay-worth 5 points)

Following the principles you learned in the Module \#5 Video, run a search (using just the P and I parts of the clinical question-don't worry about the comparison intervention or the outcome) in PubMed. Go to the "Advanced search" page and Copy your entire Search History, and paste it into the response box for this question. You will get one point for each of the following:

a) Map to the correct MeSH heading for the Patient/Problem

b) Map to the correct MeSH heading for the Intervention

c) Combine the terms properly

d) Impose the proper AGE GROUP limit

e) Limit PUBLICATION TYPES to Randomized controlled trials, Reviews, Meta-analyses, and Multicenter Studies.

Answer Feedback

A response like this one will receive full credit:

\#8 Search (\#5) AND \#6 Limits: Meta-Analysis, Randomized Controlled Trial, Review, Multicenter

Study, Adolescent: 13-18 years 21:06:25 150

\#7 Search (\#5) AND \#6 21:05:48 1911

\#6 Search "Insulin Infusion Systems"[Mesh] OR “insulin pump” 21:05:25 3288

\#5 Search insulin dependent diabetes mellitus 21:04:44 66100

Question 3 (Multiple Choice-worth 1 point)

For the remainder of this Quiz you will be working with Clinical Queries. Copy and paste the simple search (the MeSH headings for ["P" AND "I"]) into the Clinical Queries search box. What is the Category of this clinical question?

Correct Answer: Therapy

Other (incorrect) choices: Diagnosis, Prognosis, Etiology, Clinical Prediction Guides

Feedback for Incorrect Answer

Therapy is the correct category for this clinical question.

Question 4 (Essay-worth 1 point)

Run a Therapy/Narrow search for our clinical question in Clinical Queries. Copy the line with your Narrow/Specific Search and paste it here, along with the number of results. I am looking for the use of the correct P and I terms, and the correct Category and Scope. ***Also, make sure you don't still have your limits from your previous search active! This is important because I asked you to apply publication type limits in an earlier question.***

Answer Feedback

Below are two examples of responses that would receive full credit:

\#9 Search (insulin dependent diabetes mellitus AND insulin infusion systems) AND

(Therapy/Narrow[filter]) 21:28:23 198

OR

\#10 Search (insulin dependent diabetes mellitus AND "Insulin Infusion Systems"[Mesh] OR

"insulin pump”) AND (Therapy/Narrow[filter]) 21:31:41 225

Question 5 (Essay-worth 1 point)

Now, run the clinical question in Clinical Queries, as a Therapy question with a Broad Scope. 
Copy your search string and the number of results and paste it in the answer field to get credit. Make sure the number of search results is included in what you paste. Also, make sure you don't still have your limits from your previous search active! Make sure to clear your previous limits. This is important because I asked you to apply publication type limits in an earlier question.

Answer Feedback

A correct response to this question will look something like this:

\#12 Search (insulin dependent diabetes mellitus AND insulin infusion systems) AND

(Therapy/Broad[filter]) 21:44:51 1333

OR

\#11 Search (insulin dependent diabetes mellitus AND "Insulin Infusion Systems"[Mesh] OR

“insulin pump”) AND (Therapy/Broad[filter]) 21:43:34 1559

Question 6 (Essay-worth 1 point)

For the Broad/Sensitive search in Clinical Queries, use the AGE GROUP limit to target articles about our Patient's particular age group. Copy and Paste the pertinent line(s) from your Search History (on the Advanced Search page). Be sure to include the number of results.

Answer Feedback

A response that receives full credit will look like:

\#13 Search (insulin dependent diabetes mellitus AND insulin infusion systems) AND

(Therapy/Broad[filter]) Limits: Adolescent: 13-18 years 21:48:46 368

OR

\#14 Search (insulin dependent diabetes mellitus AND "Insulin Infusion Systems"[Mesh] OR

"insulin pump") AND (Therapy/Broad[filter]) Limits: Adolescent: 13-18 years 21:50:22 400 